\title{
Clinical factors associated with fatigue over time in paediatric oncology patients receiving chemotherapy
}

\author{
C-H Yeh*, , Y-C Chiang ${ }^{2}$, L Lin ${ }^{3}$, C-P Yang ${ }^{4}$, L-C Chien ${ }^{5}$, MA Weaver ${ }^{6}$ and H-L Chuang ${ }^{7}$ \\ 'Graduate Institute of Nursing Science, Chang Gung University, Kwei-San, Tao-Yuen, Taiwan; ${ }^{2}$ Department of Nursing, Chang Gung Institute of \\ Technology, Kwei-San, Tao-Yuen, Taiwan; ${ }^{3}$ School of Nursing, University of North Carolina at Chapel Hill, Chapel Hill, NC, USA; ${ }^{4}$ Department of \\ Hematology and Oncology, Chang Gung Children's Hospital, Kewi-San, Tao-Yuen, Taiwan; ${ }^{5}$ Department of Biostatistics, University of North Carolina at \\ Chapel Hill, Chapel Hill, NC, USA; ${ }^{6}$ Family Health International; ${ }^{7}$ Ten-Chen General Hospital, Taiwan
}

The purpose of this study was to investigate the relationships between clinical factors (including haemoglobin value, chemotherapeutic agents, and corticosteroid use) and changing patterns of fatigue before and for the next 10 days following the start of a new round of chemotherapy in children with cancer. A prospective longitudinal design was used to collect data from 48 paediatric oncology patients who were about to begin a new round of chemotherapy and their parents. Fatigue levels were assessed using multidomain questionnaires with three categories of patient self-report (including 'General Fatigue', 'Sleep/Rest Fatigue', and 'Cognitive Fatigue') and four categories of parent proxy-report (including 'Lack of Energy', 'Unable to Function', 'Altered Sleep', and 'Altered Mood'). The findings suggest that fatigue from both patient self-report and parent proxy-report changed significantly over time. The major findings from this study are that patients have more problems with fatigue in the first few days after the start of a cycle of chemotherapy. Corticosteroid use and haemoglobin value were associated with significant increases in fatigue that were sustained for several days and reached the highest level of fatigue at day 5 for those receiving concurrent steroids. The association of chemotherapeutic agents with fatigue varied between patient self-report and parent report, but the type of chemotherapeutic agents used was not associated with most changes in fatigue.

British Journal of Cancer (2008) 99, 23-29. doi:10.1038/sj.bjc.6604434 www.bjcancer.com

Published online 24 June 2008

(c) 2008 Cancer Research UK

Keywords: fatigue; paediatric oncology patient; clinical factors; chemotherapy; corticosteroids

Fatigue is one of the most frequent and severe symptoms experienced by paediatric oncology patients during treatment (Hockenberry-Eaton et al, 1998; Hinds et al, 1999; Wolfe et al, 2000; Collins et al, 2002). It is also among the most distressing symptoms reported in the terminal phase of their illness (Wolfe et al, 2000; Jalmsell et al, 2006; Theunissen et al, 2007). However, very few quantitative studies have reported on fatigue in paediatric oncology patients (Varni et al, 2002; Langeveld et al, 2003). Aggressive treatment of paediatric oncology patients is focused on cure; therefore, side effects, such as fatigue, that result from treatments may be ignored by clinicians (Hockenberry-Eaton and Hinds, 2000) or considered as unavoidable symptoms that need to be endured (Gibson et al, 2005).

The development of the proposed models for fatigue is based on adult oncology patients (Piper, 1986; Nail and Winningham, 1993; Wessely et al, 1998). The management of fatigue is an important clinical issue because fatigue is recognised as having a greater impact on patients than other mental or physical consequences of cancer or its treatment (Stone et al, 2000; Ahlberg et al, 2005). Fatigue is complex and is often associated with multiple physiological and emotional sequelae. Few studies have examined

\footnotetext{
*Correspondence: Professor C-H Yeh, Graduate Institute of Nursing Science, Chang Gung University, 259 Wen-Hwa I Road, Kwei-San, Tao-Yuen 33302, Taiwan; E-mail: cyeh@mail.cgu.edu.tw

Revised I May 2008; accepted 2 May 2008; published online 24 June 2008
}

the aetiology of fatigue associated with chemotherapy treatment (CTX). In a study that evaluated the epidemiology of cancerrelated fatigue in 419 adult cancer patients, $74 \%$ of the patients considered fatigue as a symptom to be endured and $80 \%$ of the oncologists believed fatigue is undertreated (Vogelzang et al, 1997). Fatigue may exist before, during, and after CTX for cancer (Langeveld et al, 2000). Stasi et al (2003) proposed that fatigue would be more intense in the first few days after CTX and then would gradually abate until the next course of CTX. However, little empirical evidence exists to support this statement.

Factors that may contribute to fatigue intensity, such as anaemia (Wang et al, 2002) and the role of cortisol (Payne, 2004), are not well documented. Corticosteroids are essential in the treatment of acute lymphoblastic leukaemia, which represents the majority of cancers diagnosed in childhood (Gaynon and Lustig, 1995). However, the relationship between fatigue and treatment protocols including the use of corticosteroids has not been investigated in detail.

Identifying the specific factors associated with the fatigue that accompanies CTX could provide important information to clinicians and researchers. Clinicians would be able to assess and treat these symptoms, not only making the children more comfortable but increasing patient and parent satisfaction as well (Braud et al, 2003). It is important for clinicians to acknowledge the impact of fatigue and, whenever possible, provide therapeutic interventions to decrease it. Thus, the purpose of this study was to investigate changing patterns of fatigue by enrolling paediatric 
oncology patients just before the start of a new round of CTX and then following them for 10 days. Factors that may contribute to fatigue, including chemotherapeutic agents, corticosteroid use during the study period, and haemoglobin value, were also examined.

\section{MATERIALS AND METHODS}

A prospective longitudinal design was used to evaluate fatigue in paediatric oncology patients who received CTX. Each subject and their parent provided data about the child's fatigue experiences before CTX and each of the next 10 days.

\section{Participants}

Participants were children with cancer and their parents. Children were eligible for this study if they were aged 7-18 years, were diagnosed with cancer, were prescribed CTX, and were not receiving concomitant radiation or had not undergone bone marrow transplantation. All children were prescribed one of the following chemotherapeutic agents: methotrexate, vincristine, etoposide, cisplatin, cytarabine, epirubicin, or cyclophosphamide. Data of concomitant corticosteroids used as part of the protocol for the CTX cycle were also collected. If a patient received corticosteroids for treatment purposes, other than a component of their CTX protocol, they were excluded from this study. Corticosteroids used included prednisolone, hydrocortisone, or dexamethasone. Owing to different CTX protocols, participants were enrolled in this study when they were about to receive a new round of CTX and their first fatigue assessment could be made on the day before the new round was started. Additional assessments were made $24 \mathrm{~h}$ after the CTX had begun (i.e., day 02) and then daily for the next 9 days. The research team selected 11 days as the timeframe to allow sufficient time for symptoms to change, as well as for administration of chemotherapeutic agents and steroids. In most CTX protocols, these agents were administered within a week, and this allowed at least 3 days to collect data on the resulting symptoms. Fifty-two patients were approached and two patients declined because of parental time constraints. Two other patients who had been on CTX for a more extended period of time (greater than 30 months) were also excluded. In total, data were collected from 48 patients and 48 parents. Table 1 presents the detailed demographic and diagnostic characteristics of the children.

Table I Demographic characteristics of patients

\begin{tabular}{|c|c|c|}
\hline Variables & $n$ & $\%$ \\
\hline \multicolumn{3}{|l|}{ Age } \\
\hline Mean (s.d.) (range) & $|1.5|(2.95)(7-17)$ & \\
\hline \multicolumn{3}{|l|}{ Gender } \\
\hline Male & 26 & 54 \\
\hline Female & 22 & 46 \\
\hline \multicolumn{3}{|l|}{ Duration since initial diagnosis (months) } \\
\hline Mean (s.d.) (range) & $4.37(4.53)(0-21)$ & \\
\hline \multicolumn{3}{|l|}{ Type of disease } \\
\hline Leukaemia & 25 & 52 \\
\hline Lymphoma & 2 & 4 \\
\hline Solid tumour & 17 & 36 \\
\hline Brain tumour & 4 & 8 \\
\hline \multicolumn{3}{|l|}{ Illness stages } \\
\hline Newly diagnosed within 2 months & 18 & 38 \\
\hline Remission on treatment & 30 & 62 \\
\hline
\end{tabular}

\section{Measures}

The conceptual definition used in this study was defined by our previous qualitative study of exploring the fatigue experiences reported by Taiwanese children with cancer (Chiang et al, 2008b). Fatigue is defined as 'a phenomenon that includes both physical and psychosocial sensations (e.g., tiredness, lack of energy, and lack of motivation) as well as consequences (e.g., lying on the bed and being unable to engage in physical activities)' (Chiang et al, $2008 \mathrm{~b}$ ). Owing to the limited number of fatigue measurement tools available at data collection, two existing instruments were used to collect data for this study. The PedQL Multidimensional Fatigue was administered to the patients and the Parent Fatigue Scale (PFS) was used for the parents. Both were originally designed and tested in English. For our studies, both measures were translated from English into Chinese language. The detailed process of translation and back translation is described in another publication (Chiang et al, 2008a).

\section{PedsQL Multidimensional Fatigue scale}

The PedsQL Multidimensional Fatigue scale (Varni et al, 2002) was used to measure patients' self-reported fatigue symptoms. It consists of three subscales, including 'General Fatigue' (six items), 'Sleep/Rest Fatigue' (six items), and 'Cognitive Fatigue' (six items). All items used a five-point Likert response set, ranging from $1=$ 'not at all' to $5=$ 'always'. To assess the detailed pattern of fatigue during chemotherapy for paediatric oncology patients, a new version of the PedsQL Multidimensional Fatigue scale, one that was intended for daily assessment of fatigue for the past $24 \mathrm{~h}$, was developed and tested (Chiang et al, 2008a). The reliabilities of the three subscales and total scale in the 24-h version ranged between 0.83 and 0.93 (Cronbach's $\alpha$ coefficient) and the validity was also evident with high correlation with the Chinese version of the Fatigue Scale-Children (Pearson correlation values ranged from 0.36 to 0.56 ) (Chiang et al, 2008a). In the original instrument, higher scores indicated fewer symptoms of fatigue. To compare the fatigue change patterns for patients and parents, the PedsQL Multidimensional Fatigue Scale used in the current study was rescored so that higher scores indicated that patients had increased fatigue. Cronbach's $\alpha$ values for the various subscales ranged from 0.91 to 0.95 for this study.

\section{The Parent Fatigue Scale}

The PFS (Hockenberry et al, 2003) was used to measure parental perception of their child's fatigue over the previous $24 \mathrm{~h}$. The PFS included four fatigue subscales: 'Lack of Energy' (six items), 'Unable to Function' (three items), 'Altered Sleep' (four items), and 'Altered Mood' (four items). All items used a five-point Likert response set, ranging from $1=$ 'not at all' to $5=$ 'always'. Higher scores indicated that parents perceived that their child experienced greater fatigue. Good reliability has been reported in previous studies with this instrument (Cronbach's $\alpha$ for the total scale was 0.88) (Hockenberry et al, 2003). Strong correlation with the child's self-report of fatigue, as well as convergent validity with other wellestablished measures, was also reported (Hockenberry et al, 2003). Cronbach's $\alpha$ values for the subscales ranged from 0.61 to 0.87 for this study.

\section{Demographic information}

Demographic and relevant data were collected from medical records and included age, gender, cancer diagnosis, haemoglobin level, body weight, and treatment information. Haemoglobin level at the baseline assessment (i.e., from blood drawn on the day before the first dose of that round of CTX) was available for all patients. For some patients $(n=17)$, haemoglobin was assessed 
only at baseline. However, the remaining patients $(n=31)$ had at least one haemoglobin assessment during follow-up. Use of chemotherapeutic agents and corticosteroids before baseline assessment and on each day during data collection was also recorded.

\section{Procedures}

The study began after receiving approval from the Human Subjects Review Committee at Chang Gung Children's Hospital in Taiwan. The hospital's established procedures for protecting confidentiality were strictly followed. The patients in the study setting who were about to receive CTX at the hospital ward and who met the sampling criteria were approached by a trained research assistant (RA). The parents received verbal and written explanations of the study and procedures and were asked about their willingness to participate in this study along with their sick child. After parental consent and child assent were obtained, they were scheduled and interviewed in person at the hospital wards by a trained interviewer. Each patient and their parent were interviewed independently by face-to-face interviews in the hospital or by telephone if the patient was no longer hospitalised at the time of data collection. The RA administered the questionnaire package in person at the hospital for the baseline assessment and collected follow-up assessment data either in person or by phone if a patient had been discharged during the data collection period. Following data collection, identity of patients and parents was removed and all identifiable materials were stored in a locked office.

Data were collected at 11 time points, baseline (pre-CTX) and daily for 10 consecutive days. The data were collected at the same time each day (i.e., in the morning before the CTX began for that day).

\section{Data analysis}

Descriptive statistics were calculated for the demographic characteristics of the patients. It was expected that the items within each subscale of fatigue could contribute differently to the underlying fatigue construct. Thus, rather than simply adding the scores for all items within a subscale, the score for each subscale was calculated using the factor scores obtained from a single-factor analysis. The ranges of scores for each subscale were converted to the range of 1-5 so that the different fatigue levels could be directly compared. A multivariate mixed-effects model (using the MIXED Procedure in SAS) (SAS Institute Inc., 2006) was used to capture the longitudinal outcome measures (modelling covariance across time) as well as the high correlation between the multidomain outcomes (modelling covariance across the multivariate observations, such as General, Sleep/Rest, and Cognitive Fatigue for patient-reported data) (Galecki, 1994).

To account for the multivariate nature of the data (multiple correlated symptoms over time), a direct-product covariance structure (UN@AR(1)), available in PROC MIXED (SAS Institute Inc., 2006), was used. Patient self-reported and parent proxyreported data were modelled separately, because the instruments used measured slightly different concepts associated with fatigue. The model included fixed effects for time, cumulative CTX agent and cumulative corticosteroid use before baseline assessment and through each study day, and current haemoglobin level. Missing current haemoglobin values were imputed using the most recently observed haemoglobin values for each patient (for 17 patients, baseline haemoglobin value was used throughout). For each study day, chemotherapeutic agent use and corticosteroid use were each coded as $0=$ 'not used' and $1=$ 'used'. Then, to examine the cumulative relationship with fatigue factors, the cumulative use of chemotherapeutic agents and corticosteroids before the baseline assessment and through each day in this cycle of CTX was calculated and used as a covariate in the model. Model effects were tested at a significance level of 0.05 . Data analyses were performed using SAS software, version 9.1.3 (SAS Institute Inc., 2006).

\section{RESULTS}

\section{Characteristics of participants}

The patients included 26 males and 22 females. The mean age of the patients was 11.51 years (range $7-17$, s.d. $=2.95$ years). Cancer diagnoses included leukaemia $(n=25)$, lymphoma $(n=2)$, solid tumour $(n=17)$, and brain tumours $(n=4)$. The average duration for receiving CTX before the current cycle was 4.37 months (range $0-21.23$, s.d. $=4.53$ months), including eight patients who were newly diagnosed and were receiving their first round of CTX; 10 had been treated for less than 2 months, 11 between 2 and 4 months, and 19 had been treated for more than 4 months. Twentysix $(54 \%)$ patients received corticosteroids. The parents included 41 mothers, 6 fathers, and 1 adult family member.

\section{Change patterns of fatigue}

Figures 1 and 2 present the unadjusted means of factor scores of fatigue for patients' self-reports and parents' proxy-reports, respectively. Patterns of change in fatigue were determined across the 11 time points. After adjusted by cumulative days of CTX, steroids used before baseline assessment, haemoglobin value, and cumulative chemotherapeutic agent and corticosteroid use for each study day, time was associated with changes in the fatigue scores $(P<0.001)$ for patient self-reported data, which indicates that patients in this study report statistically significant fatigue when the subscales of fatigue were treated as a multidomain construct. General Fatigue increased after CTX was administered to its highest level on day 2 and then gradually decreased over time. Sleep/Rest Fatigue levels also increased after CTX and reached the highest level on day 3 , but this change was not significant over 11 time points $(P=0.21)$ after controlled by other factors. Cognitive Fatigue did not change significantly over the course of the study $(P=0.95)$, although its observed change pattern appears similar to subscales of General Fatigue and Sleep/ Rest Fatigue.

For parents' proxy-reported fatigue scores, all four of the domains of fatigue changed significantly over time $(P<0.001)$. From Figure 2, the unadjusted fatigue mean scores of Lack of Energy and Unable to Function increased immediately after CTX was administered and reached the highest levels of fatigue on day 1 and then gradually decreased until day 10; however, Unable to Function had a small peak at day 5. Altered Mood reached its highest intensity level on day 5 and then decreased thereafter. Altered Sleep showed a small fluctuation until day 5, but generally decreased until day 10.

\section{Factors associated with the patterns of change in fatigue}

For patients' self-reported fatigue scores, the cumulative use of corticosteroids before baseline assessment and during study day, chemo drugs before baseline assessment, and haemoglobin were significantly associated with increased fatigue for all three subscales (i.e., General Fatigue, Sleep/Rest Fatigue, and Cognitive Fatigue). Chemotherapeutic drug administration during the study days was not associated with fatigue change.

Figure 3 compares the unadjusted mean scores of fatigue change patterns for patient self-reported data over time for those patients who ever received corticosteroids and those who did not (descriptive data for fatigue factor scores are not presented here but are available on request). Among those patients who did not receive corticosteroids, the General Fatigue, Sleep/Rest Fatigue, and Cognitive Fatigue scales decreased fairly consistently. However, for those patients who were given at least 1 day of 


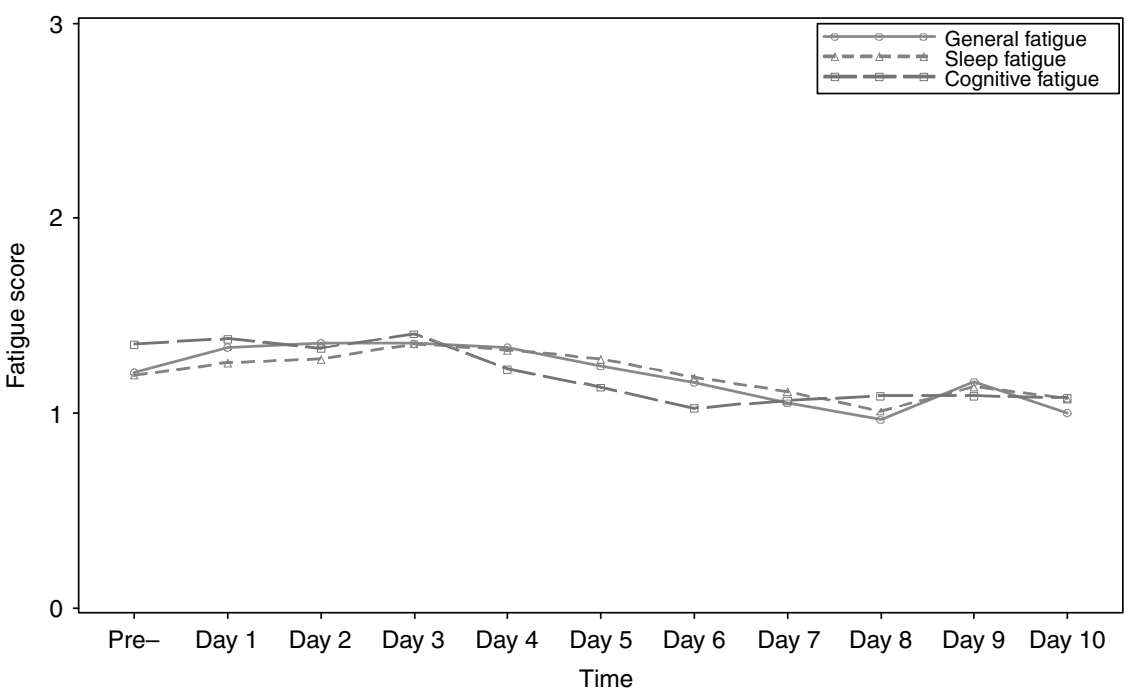

Figure I Unadjusted factor mean scores of fatigue change patterns over time reported by children.

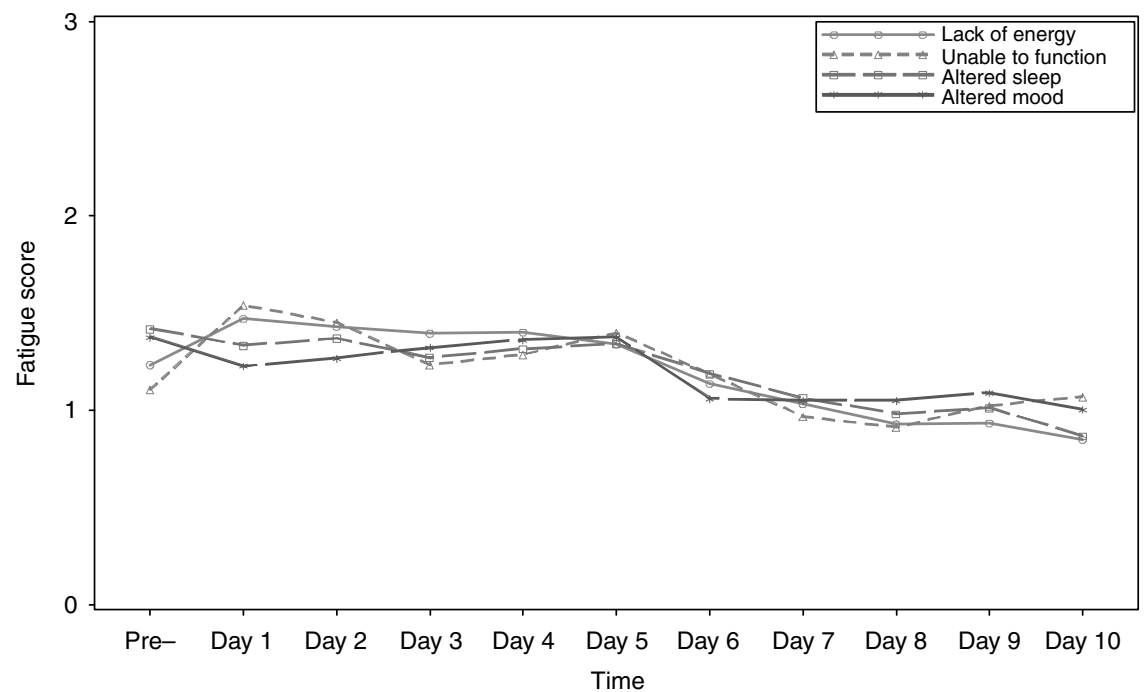

Figure 2 Unadjusted factor mean scores of fatigue change patterns over time reported by parents.

corticosteroids during this period, all three scales increased from baseline to day 3 or 4 . Day 4 or 5 had the maximum differences on the Sleep/Rest Fatigue scale. After controlling for haemoglobin and cumulative use of CTX agents, the means of all three scales were significantly higher for those using corticosteroids consecutively than for those who did not.

For parent proxy-reported data, higher levels of Lack of Energy, Altered Sleep, and Altered Mood were significantly associated with increasing cumulative corticosteroid use and a decrease in haemoglobin value $(P<0.001)$. Figure 4 shows that the unadjusted fatigue mean scores of corticosteroids appear to increase all fatigue scores in the beginning, even though the increase is very slow, besides Unable to Function, which had a sudden jump at day 1 , but decreased later, and had another jump at Day 5. In addition, before Day 2, patients who did not use corticosteroids had a higher fatigue score in Unable to Function than those without corticosteroid use. Generally speaking, all of the unadjusted fatigue mean scores reached their highest average value on day 4 or 5 for patients who received corticosteroids. After controlling for haemoglobin and cumulative use of CTX agents and corticosteroids, the mean of the Lack of Energy, Altered Sleep, and Altered Mood scores was significantly higher for those patients who used corticosteroids than for those who did not; although nonsignificant, a consistent pattern was observed for the Unable to Function scale as well.

\section{DISCUSSION}

This prospective longitudinal study is the first to document clinical factors associated with patterns of change in fatigue during a round of CTX, experienced by children with cancer in Taiwan. The major findings from this study are that patients have more problems with fatigue in the first few days after the start of a cycle of CTX. The administration of corticosteroids and haemoglobin value are associated with significant increases in fatigue that are sustained for several days and reach the highest level of fatigue at Day 5 for those using steroids consecutively. Of note, parent proxy-report data and patient self-report data indicated different factors associated with changes in fatigue. We preface the detailed 

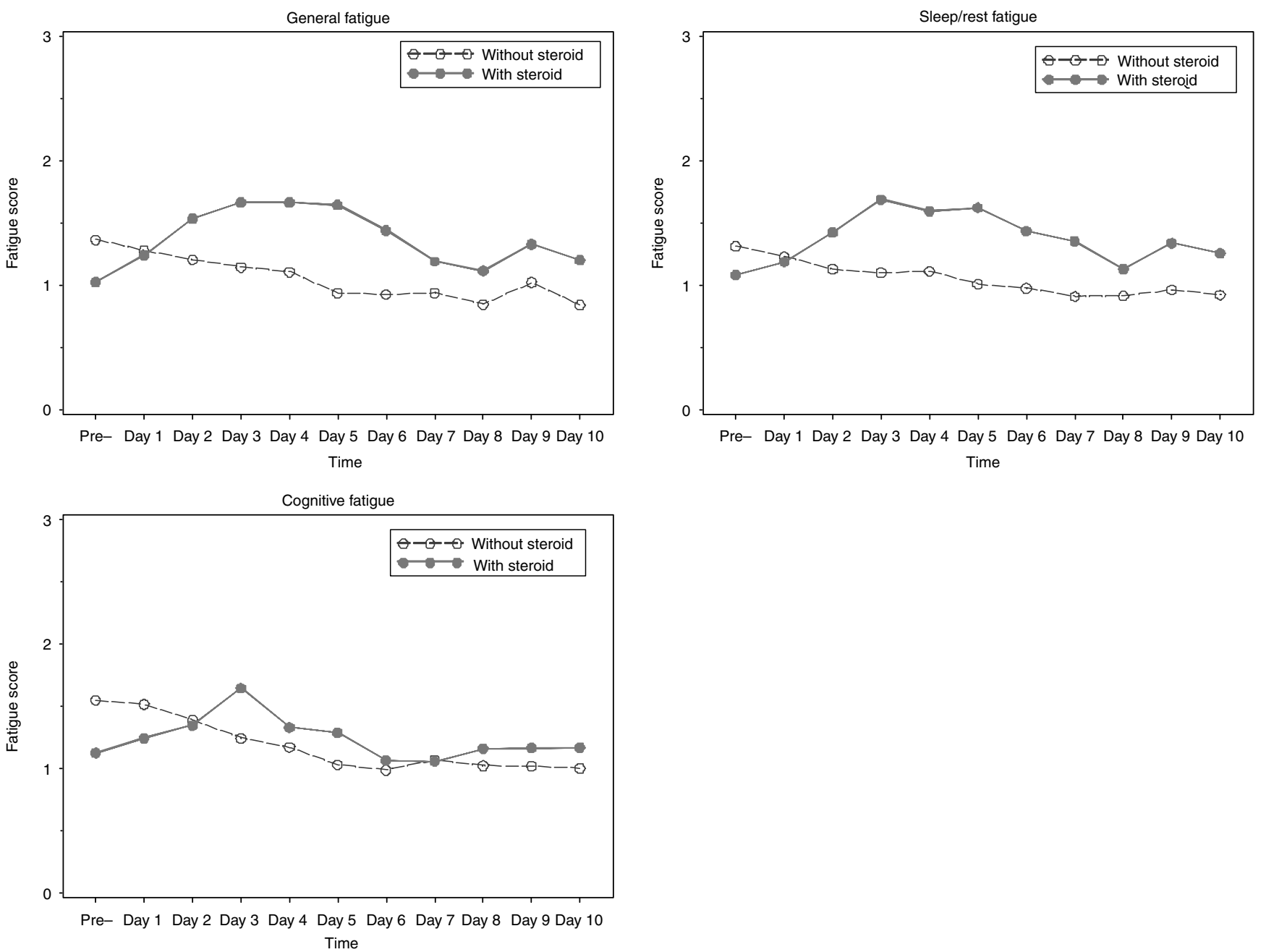

Figure 3 Unadjusted mean scores of fatigue change pattern for patients with and without corticosteroid use over time: patient self-report.

discussion of our results by listing several limitations that should be considered. First, although all children suffered from cancer and all had been receiving CTX for less than 22 months, several different CTX protocols were being used to treat the children in this study. Additionally, there were several different cancer diagnoses among the children studied. As an example, children with brain tumours may have had more pre-existing issues with fatigue than some of the other children. Lastly, all children had haemoglobin levels drawn on day 1, before starting their round of CTX; however, $35 \%$ of the children did not have subsequent haemoglobin levels drawn during the next 10 days of data collection. Another limitation of this study that may affect its interpretation is that the assessment of fatigue by patient selfreport and that by parent proxy-report are similar but showed slight variation. Thus, it is not possible to directly examine the validity of proxy-reported fatigue for patient self-reported and parent proxy-reported data. The reason for choosing these two versions is the limited established validity of the instruments in the Taiwanese population. Several authors agree that fatigue is a subjective construct (Hinds et al, 1999; National Comprehensive Cancer Network, 2006; Ream et al, 2006) and that it should be assessed directly by the patients self-report. However, the fact that children who are ill are providing these data and their cognitive and linguistic levels vary makes using only patient self-reported data less reliable (Chang and Yeh, 2005). Furthermore, brief questionnaires are needed to minimise the burden to young subjects. For example, in a study comparing quality of life measured using both child self-report and parent proxy-report (Chang and Yeh, 2005), it was determined that children younger than 12 years of age provided different perspectives of subjective domains of quality of life from parental report. In addition, when parents assess patient's fatigue, parents' own fatigue may influence their judgment of patient's fatigue.

The widely recognised definition of fatigue in paediatric oncology patients is 'profound sense of being physically tired, or having difficulty with body movement such as using their arms and legs, or opening their eyes' for children (Hockenberry et al, 2003, p 320) and 'a changing state of exhaustion that could include physical, mental and emotional tiredness' for adolescents (Hockenberry et al, 2003, p 320). The behaviours of fatigue are observable by parents and clinicians. Thus, the assessment of fatigue for paediatric oncology patients needs to take into account parent proxy-report. Parents know their children better than anyone else, and should be listened to when stating concerns about their child's fatigue. There is no 'gold standard' for fatigue assessment in children and there are few studies, even in English, that report on the validity of children's self-report data on fatigue. Thus, we collected patient self-reported and parent proxy-reported data simultaneously, to provide additional data on fatigue, particularly the data collected from younger children.

This study is the first to document the relationship between corticosteroids and fatigue. Corticosteroid use was associated with 

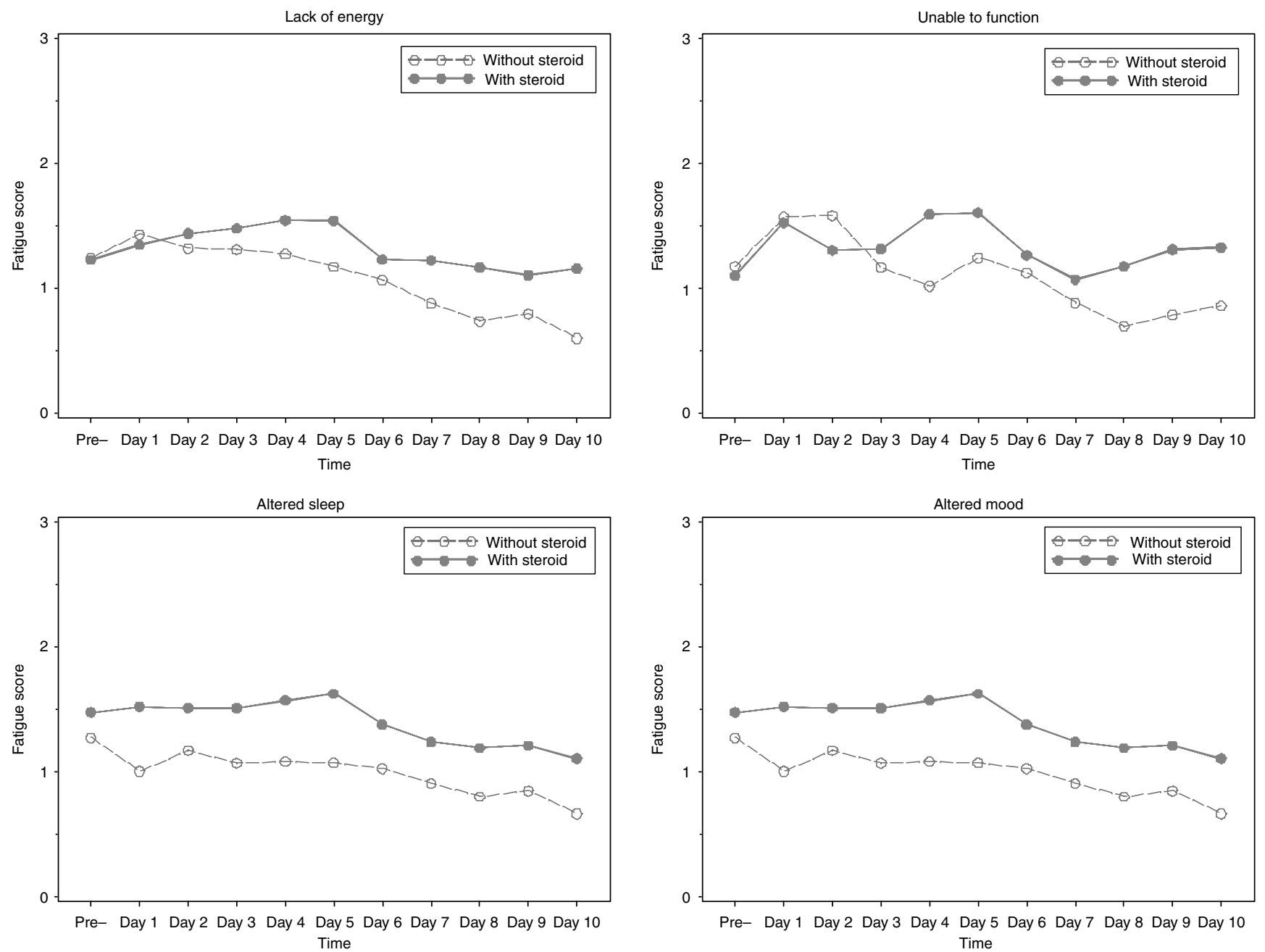

Figure 4 Unadjusted mean scores of fatigue change pattern for patients with and without corticosteroid use over time: parent proxy-report.

fatigue in most domains reported by either patients or parents. Most of the literature pertaining to adults have documented the impact of chemotherapeutic agent use on fatigue, but there was no literature to explain the relationship between corticosteroid use and fatigue in children receiving chemotherapy.

Corticosteroid use was associated with higher fatigue scores and reached the highest at day 5 . The mechanism for the corticosteroidrelated fatigue found in this study is not clear. Increased fatigue levels associated with the use of corticosteroids are in conflict with the theory of hypothalamic-pituitary-adrenal axis in which fatigue is assumed to be related to low circulating levels of cortisol (Jones et al, 1998; Payne, 2004). Patients in this study received long term and high doses of corticosteroids, which may lead to complications of corticosteroid use, such as sleep disruption, and therefore increasing the possibility of fatigue. Further study is needed to examine other possible factors that may cause fatigue, such as different cancer diagnoses, CTX agents, and pre-existing sleep problems.

The patients included in this study received a variety of CTX protocols, combining different chemotherapeutic agents, with or without corticosteroids. Owing to the sample size of this study, we did not analyse the relationship between fatigue levels and the use of corticosteroids with specific chemotherapeutic agents. The underlying mechanism of fatigue is not yet clear (Cleeland et al, 2003); this makes it difficult to develop scientific foundations for intervention to relieve fatigue.

As the sample size used is small, generalising these results without further study would be premature. However, this study can be used by clinicians and researchers as a stimulus to conduct more clinical assessments and conduct replications and expansions of the present study. Clinicians can be more vigilant about assessing fatigue and noting changes in the child's fatigue level as it relates to length of time since CTX and steroids were administered. They could also note how fatigue relates to any nausea, vomiting, or pain as well as changes in laboratory values. Clinicians could also record the parent's perception of their child's fatigue. Researchers could expand this study by recruiting more children who had the same diagnosis and CTX protocols. To conduct such studies it might require collecting data from different places.

Our study supports the assumption that lower haemoglobin level is a significant contributor to fatigue as previously described in the literature (Shafqat et al, 2005). Routine clinical laboratory check for haemoglobin in the current study setting varies, ranging from 3 days to 1 month, depending on a patient's treatment protocol. The study findings do indicate that fatigue is likely to 
vary over the course of time and treatment and that the timing of haemoglobin measurements may not be frequent enough to catch the rapidly changing fatigue level. Although the literature does not report a conclusive relationship between haemoglobin and fatigue (Hockenberry et al, 2003), this finding does suggest that frequent haemoglobin check might increase the clinicians' understanding of fatigue that accompanies CTX.

\section{ACKNOWLEDGEMENTS}

This research was supported by a grant to Dr Yeh from the National Health Research Institutes, Taiwan (Grant number NHRIEX95-9302PI) and National Science Council (NSC94-2314-B-182014). We thank Dr Merle Mishel for her thoughtful comments and Dr Susan Jay for editing the manuscript.

\section{REFERENCES}

Ahlberg K, Ekman T, Gaston-Johansson F (2005) Fatigue, psychological distress, coping resources, and functional status during radiotherapy for uterine cancer. Oncol Nurs Forum 32: 633-640

Braud AC, Genre D, Leto C, Nemer V, Cailhol JF, Macquart-Moulin G, Maraninchi D, Viens P (2003) Nurses' repeat measurement of chemotherapy symptoms: feasibility, resulting information, patient satisfaction. Cancer Nurs 26: $468-475$

Chang PC, Yeh CH (2005) Agreement between child self-report and parent proxy-report to evaluate quality of life in children with cancer. Psychooncology 14: 125-134

Chiang YC, Hinds PS, Yeh CH, Yang CP (2008a) Development and psychometric testing of a Chinese version of the Fatigue Scale-Children in Taiwan. J Clin Nurs 17: $1201-1210$

Chiang YC, Yeh CH, Wang KWK, Yang CP (2008b) The experience of cancer-related fatigue in Taiwanese children. Eur J Cancer Care (in press)

Cleeland CS, Bennett GJ, Dantzer R, Dougherty PM, Dunn AJ, Meyers CA, Miller AH, Payne R, Reuben JM, Wang XS, Lee BN (2003) Are the symptoms of cancer and cancer treatment due to a shared biologic mechanism? A cytokine-immunologic model of cancer symptoms. Cancer 97: 2919-2925

Collins JJ, Devine TD, Dick GS, Johnson EA, Kilham HA, Pinkerton CR, Stevens MM, Thaler HT, Portenoy RK (2002) The measurement of symptoms in young children with cancer: the validation of the memorial symptom assessment scale in children aged 7-12. J Pain Symptom Manage 23: $10-16$

Galecki AT (1994) General class of covariance structures for two or more repeated factors in longitudinal data analysis. Commun Statist Theory Methods 23: $3105-3119$

Gaynon PS, Lustig RH (1995) The use of glucocorticoids in acute lymphoblastic leukemia of childhood. Molecular, cellular, and clinical considerations. J Pediatr Hematol Oncol 17: 1-12

Gibson F, Garnett M, Richardson A, Edwards J, Sepion B (2005) Heavy to carry: a survey of parents' and healthcare professionals' perceptions of cancer-related fatigue in children and young people. Cancer Nurs 28: 27-35

Hinds PS, Hockenberry-Eaton M, Gilger E, Kline N, Burleson C, Bottomley S, Quargnenti A (1999) Comparing patient, parent, and staff descriptions of fatigue in pediatric oncology patients. Cancer Nurs 22: 277-288

Hockenberry MJ, Hinds PS, Barrera P, Bryant R, Adams-McNeill J, Hooke C, Rasco-Baggott C, Patterson-Kelly K, Gattuso JS, Manteuffel B (2003) Three instruments to assess fatigue in children with cancer: the child, parent and staff perspectives. J Pain Symptom Manage 25: 319-328

Hockenberry-Eaton M, Hinds PS (2000) Fatigue in children and adolescents with cancer: evolution of a program of study. Semin Oncol Nurs 16: 261-272

Hockenberry-Eaton M, Hinds PS, Alcoser P, O’Neill JB, Euell K, Howard V, Gattuso J, Taylor J (1998) Fatigue in children and adolescents with cancer. J Pediatr Oncol Nurs 15: 172-182

Jalmsell L, Kreicbergs U, Onelov E, Steineck G, Henter JI (2006) Symptoms affecting children with malignancies during the last month of life: a nationwide follow-up. Pediatrics 117: 1314-1320

Jones TH, Wadler S, Hupart KH (1998) Endocrine-mediated mechanisms of fatigue during treatment with interferon-alpha. Semin Oncol 21: 54-63
Langeveld N, Ubbink M, Smets E (2000) 'I don't have any energy': the experience of fatigue in young adult survivors of childhood cancer. Eur J Oncol Nurs 4: 20-28

Langeveld NE, Grootenhuis MA, Voute PA, de Haan RJ, van den Bos C (2003) No excess fatigue in young adult survivors of childhood cancer. Eur J Cancer 39: 204-214

Nail LM, Winningham ML (1993) Fatigue. In Cancer Nursing: Principles and Practice, Groenwald SL, Forgge MH, Goodman M, Yarbro CH (eds) 3rd edn, pp 608-619. Jones and Bartlett: Boston

National Comprehensive Cancer Network (2006) NCCN practice guidelines for cancer-related fatigue. http://www.nccn.org/professionals/physician_gls/ PDF/fatigue.pdf accessed 19 November 2006

Payne JK (2004) A neuroendocrine-based regulatory fatigue model. Biol Res Nurs 6: $141-150$

Piper BF (1986) Fatigue. In Pathophysiological Phenomena in Nursing: Human Responses to Illness, Carrieri V, Lindsey AM, West CM (eds) pp 219-234. Saunders: Philadelphia

Ream E, Gibson F, Edwards J, Seption B, Mulhall A, Richardson A (2006) Experience of fatigue in adolescents living with cancer. Cancer Nurs 29: $317-326$

SAS Institute Inc. (2006) SAS OnlineDoc ${ }^{\mathbb{R}}$ 9.1.3. SAS Institute Inc.: Cary, NC Shafqat A, Einhorn LH, Hanna N, Sledge GW, Hanna A, Juliar BE, Monahan P, Bhatia S (2005) Screening studies for fatigue and laboratory correlates in cancer patients undergoing treatment. Ann Oncol 16: 1545-1550

Stasi R, Abriani L, Beccaglia P, Terzoli E, Amadori S (2003) Cancer-related fatigue: evolving concepts in evaluation and treatment. Cancer 98: $1786-1801$

Stone P, Richards M, A'Hern R, Hardy J (2000) A study to investigate the prevalence, severity and correlates of fatigue among patients with cancer in comparison with a control group of volunteers without cancer. Ann Oncol 11: $561-567$

Theunissen JM, Hoogerbrugge PM, van Achterberg T, Prins JB, VernooijDassen MJ, van den Ende CH (2007) Symptoms in the palliative phase of children with cancer. Pediatr Blood Cancer 49: 160-165

Varni JW, Burwinkle TM, Katz ER, Meeske K, Dickinson P (2002) The PedsQL in pediatric cancer: reliability and validity of the pediatric quality of life inventory generic core scales, multidimensional fatigue scale, and cancer module. Cancer 94: 2090-2106

Vogelzang NJ, Breitbart W, Cella D, Curt GA, Groopman JE, Horning SJ, Itri LM, Johnson DH, Scherr SL, Portenoy RK (1997) Patient, caregiver, and oncologist perceptions of cancer-related fatigue: results of a tripart assessment survey. The Fatigue Coalition. Semin Hematol 34: 4-12

Wang XS, Giralt SA, Mendoza TR, Engstrom MC, Johnson BA, Peterson N, Broemeling LD, Cleeland CS (2002) Clinical factors associated with cancer-related fatigue in patients being treated for leukemia and nonHodgkin's lymphoma. J Clin Oncol 20: 1319-1328

Wessely S, Hotopf M, Sharpe M (1998) Chronic Fatigue and its Syndrome, 1 st edn. Oxford University Press: Oxford

Wolfe J, Grier HE, Klar N, Levin SB, Ellenbogen JM, Salem-Schatz S, Emanuel EJ, Weeks JC (2000) Symptoms and suffering at the end of life in children with cancer. $N$ Engl J Med 345: 326-333 